que encontrarlo en la quinta dimensión de Hofstede: "Long Term Orientation versus Short Term Normative Orientation ${ }^{(1)}$ ". Los alemanes en representación de una sociedad con previsión de futuro y planificación de vida a largo plazo, podrían poseer una tradición de contratación de numerosos seguros que eviten altos riesgos.

Celia L. Vázquez concluye este bloque con su artículo "Trabajar como enfermero español en un hospital universitario de Erlangen". En el mismo nos presenta el proyecto llevado a cabo en este hospital en 2012 para acoger a 27 enfermeros españoles describiéndonos sus experiencias interculturales. En el ámbito de la salud también existen diferencias entre ambos países, especialmente en las competencias de un facultativo alemán y en una más rígida jerarquía en el sistema hospitalario.

En el bloque relacionado con el mundo estudiantil, María Eugenia de la Torre, nos presenta las diferencias existentes en los sistemas educativos de ambos países. Las más destacables hay que encontrarlas en la relación profesor-alumno (más jerárquica en Alemania), en los contenidos de las asignaturas (más generales en España) y los criterios de evaluación.
Laia Àlvarez nos relata algunas experiencias interculturales de españoles en Berlín. Las principales diferencias las observa, al igual que cada uno de nosotros que haya tenido una experiencia Erasmus en Alemania, en el modo y estilo de vida, en las características de las fiestas estudiantiles (en Alemania más estrictas en cuanto a las invitaciones), la moda, la privacidad del espacio personal, la puntualidad y el control de los billetes en los medios de transporte y por último en la actitud ante el reciclaje.

Estas experiencias se contrastan en el siguiente artículo, con las del propio autor como estudiante alemán en Barcelona, quien ve en los españoles una clara diferencia en relación a las invitaciones, a la formas de quedar y especialmente a la actitud frente al tiempo para la consecución de proyectos.

De gran interés me resulta el artículo de Jürgen Bolten, “QQué es competencia intercultural?”. En el mismo nos constata la ausencia de una definición de aceptación general al respecto y defiende la idea de que la formación intercultural que se ha venido llevando a cabo tradicionalmente desde los años 50, no puede ya aplicarse a los nuevos contextos.

“La capacitación no es un constructo universal, sino que está estrechamente ligada al contexto [...] $\mathrm{La}$ identidad se constituye en la interconexión con los demás" (págs. 267-268). Por ello, deberíamos reflexionar en lo que respecta a la enseñanza de habilidades interculturales, si no sería mejor, en vez instruir conocimientos universales para superar situaciones problemáticas y malentendidos, utilizar las sinergias a través de las interconexiones culturales.

E. Holenstein y Yokohama finalizan el bloque y concluyen la obra con su artículo "Una docena de reglas de buen cubero para evitar malentendidos culturales".

Es un artículo que se aproxima a la contrastación cultural entre Europa y Japón, más que a Alemania y España. Aunque se aleja del objetivo específico de responder a la pregunta del título del libro, nos invita a todos aquellos que nos encontramos trabajando en un proyecto de investigación de contrastación cultural, a tener en cuenta doce reglas. Todas ellas poseen un denominador común: el principio de equidad. En este sentido, debemos ser tolerantes, ponernos en el en lugar del otro, evitar juicios de valor, tener en cuenta principios objetivos y evitar las dualidades; $y$ por último, comprender que no todo es comprobable científicamente.
Finalmente, podemos concluir que la obra, a pesar de estar aparentemente inconexa en su estructura y no cumplir con el objetivo de proporcionarnos las claves para conseguir una comunicación hispano-alemana de éxito en cada uno de sus artículos, es claramente recomendable su lectura, puesto que permite al lector obtener una primera aproximación general sobre los aspectos a tener en cuenta en la contrastación cultural.

Raquel Mateo González

Universidad Pablo de Olavide Sevilla

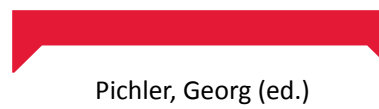

Extremos. Visiones de lo extremo en literatura, historia, música, arte, cine $y$ linguística en España y Austria

Berna (Suiza), Peter Lang, 2017, 390 págs., ISBN: 9783034326896 http://dx.doi.org/10.12795/ mAGAzin.2016.i24.07

Extremos. Visiones de lo extremo en literatura, historia, música, arte, cine y lingüística en España y Austria contiene un total de 18 textos escritos por investigadores de distintos ámbitos del mundo de las Humanidades y que han sido recopilados por el 
profesor de la Universidad de Alcalá Georg Pichler. Los escritos incluidos en este tomo de la colección "Perspektiven der Germanistik und Komparatistik in Spanien" de la editorial Peter Lang, tuvieron su origen en una serie de ponencias celebradas durante el XIV Simposio Austro-Hispano en la Universidad de Alcalá en mayo de 2014. "Extremos", la palabra que da título a esta obra colectiva, se erige como el eje vertebrador que recorre todos los artículos, dando una visión caleidoscópica de distintas manifestaciones humanísticas españolas y austriacas durante el siglo XX.

La primera sección, a la que más espacio se le dedica en el tomo, aborda temas relacionados con el campo de la lingüística y la traducción. Abre este bloque el texto de Raúl Sánchez Prieto sobre las diferentes maneras de afrontar los conflictos ideológicos entre el idioma dominante y las lenguas regionales dentro de un mismo estado, como es el caso del catalán valenciano y el gallego en España, y el esloveno en la región austriaca de Carintia. También siguiendo la influencia de la política en la lengua, Paul Danler compara el uso del pathos retórico en los discursos de unificación nacional dados por Franco el 19 de abril de 1937, y por Hitler el 15 de marzo de 1938 en España y Austria respectivamente. Los dos artículos si- guientes, por Marta Fernández-Villanueva y José Javier Martos Ramos, versan sobre el empleo del lenguaje en los medios de comunicación. El de la primera se sirve de programas televisivos en España y Austria para analizar la descortesía lingüística y sus efectos en los interlocutores implicados. Por su parte, Martos Ramos estudia las distintas maneras en las que la interacción periodistalector se lleva a cabo en noticias austriacas sobre España. Asimismo, destacan los artículos de Cornelia Feyrer sobre las diferentes formas que toma el concepto de "lo extremo" en los discursos médicos y el de María José Jiménez Vázquez, que muestra cómo Internet ha desdibujado las fronteras entre lexicógrafos y usuarios de diccionario con la aparición de diversos recursos online. Cerrando este apartado, Wolfgang Pöckl se adentra en las estrategias que emplea el austriaco H.C. Artmann para mantener la estética "extrema” de Quevedo, caracterizada así por Rafael Lapesa, en la versión austriaca de El Buscón.

La segunda sección explora manifestaciones extremas en las artes visuales y la música. El primer texto, por Christopher F. Laferl, establece paralelismos y diferencias en las primeras obras de los aclamados directores Pedro Almodóvar y Ulrich Seidl. Estos no solo se adentran en temas tabúes, como la representación de la violencia y la sexualidad, desde perspectivas radicalmente opuestas, sino que también establecen relaciones "extremas" entre el contenido y la estructura técnica empleada en sus obras. Por su lado, Markus Neuwirth realiza un extenso recorrido por la vida y obra del pintor Rudolph Charles von Ripper, quien vivió en una época convulsa, marcada por los horrores del nazismo, y cuya experiencia quedó reflejada en sus pinturas. Asimismo, cabe señalar que Neuwirth complementa el contenido de su artículo con dos anexos, en los que se recogen el intercambio epistolar de von Ripper con otros intelectuales en lengua alemana de la época, como Hermann Broch y Klaus Mann. Para concluir, los artículos de Joaquín Rubio Tovar y Paloma Ortiz-deUrbina analizan las corrientes musicales que se suceden en Austria y España a finales del siglo XIX y principios del siglo XX. El primero refleja cómo la situación de la música en España y Austria es diametralmente opuesta, pues mientras que Viena estaba dando sus primeros pasos en la búsqueda de la modernidad, a España aún le quedaba un largo camino por recorrer. Por su parte, Ortiz-de-Urbina, analiza las relaciones que se establecieron entre el austriaco Arnold Schönberg, fundador del Dodecafonismo, y el español Roberto Gerhard a raíz de la Primera Guerra
Mundial, así como la influencia de este devenir histórico tanto en la creación de sus obras como en la recepción de las mismas.

La tercera sección versa sobre literatura austriaca y española, y en ella se incluyen artículos cuyas temáticas tratan la experiencia del exilio, la escritura bajo la dictadura, o el recuerdo de esta. Abre el bloque el artículo de Michael Rössner sobre el humor en Enrique Jardiel Poncela y Fritz von Herzmanovsky-Orlando, escritores que tuvieron que experimentar alternativas al humor convencional para sobrepasar la censura de los regímenes fascistas. Según argumenta Rössner, ambos autores tomaron rumbos muy similares, dentro de sus diferencias, haciendo de sus obras una hipérbole desordenada cuyo resultado solo pudo ser una risa extrema. A continuación, Patricia Cifre-Wibrow analiza cómo los acontecimientos históricos de los primeros años del siglo XX (nazismo, austrofascismo, franquismo, guerras civiles, la Segunda Guerra Mundial, Holocausto, exilio...) tuvieron un fuerte impacto sobre la pluma y la mentalidad de los escritores y pensadores Max Aub, Jean Améry y Hannah Arendt. A través de diversos textos de estos intelectuales, explora cómo estas figuras reflexionaron sobre conceptos como identidad, nacionalidad, frontera o lengua. Por su lado, Manuel Mon- 
tesinos Caperos parte de la obra austriaca Graubart Boulevard, de Christoph W. Bauer; y de la española ¿Qué fue de Joaquín Heredia?, de José Luis Gavilanes Laso para determinar las similitudes y las diferencias estéticas y discursivas que conforman ambos textos. Estos reflejan la vida de Richard Graubart y Joaquín Heredia, figuras que vivieron bajo el terror nacionalsocialista $y$ franquista $y$ fueron perseguidos por su condición de judío, el primero, y de masón, el segundo. Finalizando esta sección, los textos de Margarita Blanco Hölscher y Wolfram Krömer aportan la Weltanschauung femenina de la segunda mitad del siglo XX. La primera realiza un análisis exhaustivo del papel de las obras de las escritoras Carmen Laforet, Carmen Martín Gaite, Ingeborg Bachmann y Marlen Haushofer durante la dictadura franquista, en el caso de las españolas, y tras la Segunda Guerra Mundial, en el caso de las austriacas. En cuanto al texto de Krömer, este recoge cómo la autora catalana Maria Barbal y la

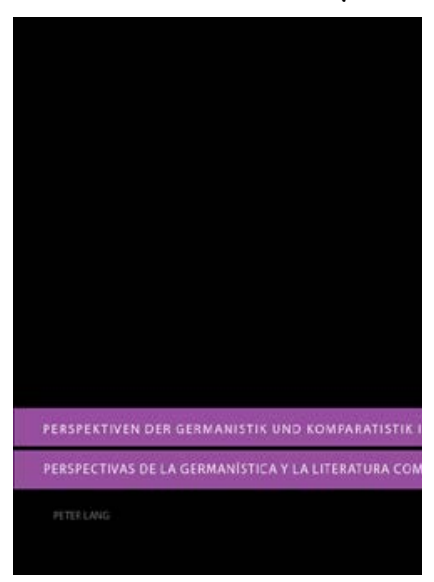

carintia Maja Haderlap reflejan en sus obras el proceso de homogeneización y exterminio cultural de las minorías catalanas y eslovenas perpetrados por las dictaduras de Franco y de Hitler en suelo español y austriaco.

El tomo finaliza con una sección dedicada al campo de la historiografía en el que los investigadores Linda Erker y Georg Pichler reflexionan sobre los puntos comunes y divergentes que han caracterizado la idiosincrasia ideológica del austrofascismo y el franquismo. Por un lado, Erker plantea la necesidad de iniciar nuevas investigaciones en torno a las relaciones entre violencia y fascismo, pues se tiende a aislar los fascismos ibéricos y a invisibilizar el austrofascismo. Por ello, centra su estudio en el papel de la violencia en los entornos universitarios del Madrid del primer franquismo y de la Viena de Dollfuß, examinando la actuación de los distintos agentes que participaron en la represión y depuración de las instituciones de enseñanza superior. Asimismo, se observan similitudes a la hora de disolver y reconfigurar un sistema educativo acorde a la nueva ideología totalitaria. Por su parte, el profesor Pichler cuestiona la validez de los acercamientos tradicionales a la teoría del fascismo a la hora de afrontar el estudio del franquismo y del austrofascismo. Según el autor, estas aproximaciones se caracterizan por su generalidad y su incapacidad para ofrecer herramientas eficientes que permitan analizar de forma específica fascismos periféricos. Para solventar dicha problemática, plantea un análisis comparativo entre los casos español y austriaco, en el que se enfatizan aspectos tales como la historiografía, la estructuración sociopolítica del estado, la operatividad de la violencia y la represión, y la recepción de la memoria histórica.

En conclusión, este volumen abre la puerta a investigaciones ulteriores en torno a la gran variedad de temas abordados. Debido a la especificidad del contenido, las aportaciones realizadas por los investigadores participantes en el mismo pueden suscitar un gran interés para el mundo académico. Es interesante remarcar cómo se ha conceptualizado la noción de "lo extremo", ya que esta es la que guía la perspectiva comparada de esta obra. Partiendo de la caracterización que hace el historiador británico Eric Hobsbawm en su obra Age of the Extremes, los 18 artículos aquí reunidos analizan cómo se dan estos extremos en diferentes manifestaciones humanísticas de España y Austria producidas durante el "corto siglo XX". Así, campos como el de la lingüística, las artes, la literatura o la historiografía quedan ligados por un solo hilo conductor: lo extremo.

A su vez, cabe señalar que "lo extremo" no solo se en- cuentra en el contenido de los temas tratados, sino que alcanza niveles metatextuales, pues al plasmarlo desde ámbitos tan aparentemente distantes entre sí, como la lingüística y la historiografía, se genera una dinámica "extrema" entre los diversos artículos. Extremos. Visiones de lo extremo en literatura, historia, música, arte, cine y lingüística en España y Austria es, por todo esto, un ejemplar de gran utilidad para ampliar nuevos horizontes en estas ramas del conocimiento, incorporando "lo extremo" como elemento de interés analítico.

Patricia Pizarroso Acedo y Alejandro Rivero/Vadillo

Universidad de Alcalá

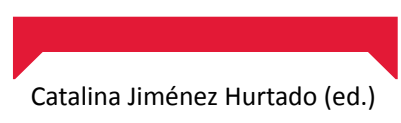

\section{La traducción} como comunicación
interlingǘística
transcultural
mediada

Frankfurt, Peter Lang 2015. Selección de artículos de Gerd Wotjak.

Con el volumen titulado La traducción como comunicación interlingüística transcultural mediada nos hallamos ante un tipo infrecuente pero sumamente interesante de compilación: Catalina Jiménez Hurtado, catedrática de Traducción de la Universidad de Granada, 